\title{
Quantized coexisting electrons and holes in graphene measured using temperature-dependent magnetotransport
}

Citation for published version (APA):

Kurganova, E. V., Wiedmann, S., Giesbers, A. J. M., Gorbachev, RV., Novoselov, KS., Katsnelson, M. I., Tudorovskiy, T., Maan, J. C., \& Zeitler, U. (2013). Quantized coexisting electrons and holes in graphene measured using temperature-dependent magnetotransport. Physical Review B, 87(8), 085447-1/5. https://doi.org/10.1103/PhysRevB.87.085447

DOI:

10.1103/PhysRevB.87.085447

Document status and date:

Published: 01/01/2013

\section{Document Version:}

Publisher's PDF, also known as Version of Record (includes final page, issue and volume numbers)

\section{Please check the document version of this publication:}

- A submitted manuscript is the version of the article upon submission and before peer-review. There can be important differences between the submitted version and the official published version of record. People interested in the research are advised to contact the author for the final version of the publication, or visit the $\mathrm{DOI}$ to the publisher's website.

- The final author version and the galley proof are versions of the publication after peer review.

- The final published version features the final layout of the paper including the volume, issue and page numbers.

Link to publication

\section{General rights}

Copyright and moral rights for the publications made accessible in the public portal are retained by the authors and/or other copyright owners and it is a condition of accessing publications that users recognise and abide by the legal requirements associated with these rights.

- Users may download and print one copy of any publication from the public portal for the purpose of private study or research.

- You may not further distribute the material or use it for any profit-making activity or commercial gain

- You may freely distribute the URL identifying the publication in the public portal.

If the publication is distributed under the terms of Article 25fa of the Dutch Copyright Act, indicated by the "Taverne" license above, please follow below link for the End User Agreement:

www.tue.nl/taverne

Take down policy

If you believe that this document breaches copyright please contact us at:

openaccess@tue.nl

providing details and we will investigate your claim. 


\title{
Quantized coexisting electrons and holes in graphene measured using temperature-dependent magnetotransport
}

\author{
E. V. Kurganova, ${ }^{1,}{ }^{*}$ S. Wiedmann, ${ }^{1}$ A. J. M. Giesbers, ${ }^{2}$ R. V. Gorbachev, ${ }^{3}$ K. S. Novoselov, ${ }^{3}$ M. I. Katsnelson, ${ }^{4}$ \\ T. Tudorovskiy, ${ }^{4}$ J. C. Maan, ${ }^{1}$ and U. Zeitler ${ }^{1, \dagger}$ \\ ${ }^{1}$ High Field Magnet Laboratory, Institute for Molecules and Materials, Radboud University Nijmegen, Toernooiveld 7 , \\ 6525 ED Nijmegen, The Netherlands \\ ${ }^{2}$ Department of Applied Physics, Eindhoven University of Technology, P.O. box 513, 5600 MB Eindhoven, The Netherlands \\ ${ }^{3}$ School of Physics and Astronomy, University of Manchester, M13 9PL Manchester, United Kingdom \\ ${ }^{4}$ Institute for Molecules and Materials, Radboud University Nijmegen, Heyendaalseweg 135, 6525 AJ Nijmegen, The Netherlands
}

(Received 20 November 2012; published 28 February 2013)

\begin{abstract}
We present temperature-dependent magnetotransport experiments around the charge neutrality point in graphene and determine the amplitude of potential fluctuations $s$ responsible for the formation of electron-hole puddles. The experimental value $s \approx 20 \mathrm{meV}$ is considerably larger than in conventional semiconductors, which implies a strong localization of charge carriers observable up to room temperature. Surprisingly, in the quantized regime, the Hall resistivity overshoots the highest plateau values at high temperatures. We demonstrate by model calculations that such a peculiar behavior is expected in any system with coexisting electrons and holes when the energy spectrum is quantized and the carriers are partially localized.
\end{abstract}

DOI: 10.1103/PhysRevB.87.085447

\section{INTRODUCTION}

Pristine graphene is a zero-gap semiconductor where the conduction and valence band touch at zero energy. This point is generally referred to as the charge neutrality point (CNP) with a zero charge carrier density. However, it has been shown experimentally that for graphene on $\mathrm{Si} / \mathrm{SiO}_{2}$ substrates, electrons and holes coexist around the CNP, which is attributed to the presence of spatially inhomogeneous conducting electron-hole puddles. ${ }^{1-4}$

In this paper we investigate the coexistence of electrons and holes by means of temperature-dependent magnetotransport around the CNP. We present results in the classical and quantum Hall regimes. In the classical regime, the individual charge carrier concentration and conductivity at the CNP increases with increasing temperature due to thermal activation but as a consequence of potential fluctuations, do not vanish even at the lowest temperature. From this temperature dependence, we determine the strength of the potential fluctuations responsible for the formation of electron-hole puddles, $s \approx$ $20 \mathrm{meV}$ for our samples. In the quantum Hall regime, the Hall resistance shows higher values than expected, suggesting a deactivation of charge carriers with increasing temperature. Using model calculations, however, we demonstrate that this counterintuitive temperature dependence is straightforwardly explained considering the quantized density of states of coexisting electrons and holes.

The paper is organized as follows: In Sec. II we shortly describe the samples an measurements. Section III is devoted to the experiential magnetotransport in the classical regime along with an interpretation of the results. In Sec. IV we describe experimentally (Sec. IV A) and discuss theoretically (Sec. IV B) magnetotransport data in the quantum Hall regime. The paper ends with a conclusion in Sec. V.

\section{EXPERIMENT}

We have measured two field effect transistors made from single-layer graphene (SLG) and bilayer graphene (BLG) deposited on $\mathrm{Si} / \mathrm{SiO}_{2}$ wafers and shaped into a $1-\mu \mathrm{m}$-wide Hall bar. Both flakes originate from natural graphite and have mobilities $\mu=1 \mathrm{~m}^{-2} \mathrm{~s}^{-1}$ and $\mu=0.3 \mathrm{~m}^{-2} \mathrm{~s}^{-1}$, respectively. The total charge carrier concentration $n$ is controlled by applying a gate voltage $V_{g}$ on the conducting Si substrate: ${ }^{5}$

$$
n=n_{e}-n_{h}=\alpha V_{g},
$$

with $\alpha=7.2 \times 10^{14} \mathrm{~m}^{-2} / \mathrm{V}$ for a 300 -nm-thick $\mathrm{SiO}_{2}$ gate insulator. The subscripts $e$ and $h$ indicate electrons and holes, respectively.

Magnetotransport as a function of magnetic field, carrier concentration, and temperature were performed in a temperature range between 0.5 and $250 \mathrm{~K}$ using a top-loading He-3 system and a variable-temperature insert in a $33 \mathrm{~T}$ Bitter magnet.

\section{CLASSICAL REGIME}

Figure 1(a) shows $\rho_{x y}(n)$ for the BLG sample at $B=0.8 \mathrm{~T}$ for different temperatures. Down to the lowest temperatures, the Hall resistivity does not exhibit quantized plateaus at this magnetic field, indicating a continuous energy spectrum. For all temperatures, the Hall resistance smoothly crosses zero at the CNP, which indicates that electrons and holes are present at all gate voltages. ${ }^{6}$

In this coexistence regime, the simultaneous contribution of electrons and holes with equal mobility $\mu$ to the Hall effect is given by ${ }^{7,8}$

$$
\rho_{x y}=\frac{B}{e} \frac{\left(n_{e}-n_{h}\right)\left(1+(\mu B)^{2}\right)}{\left(n_{e}+n_{h}\right)^{2}+\left[\left(n_{e}-n_{h}\right) \mu B\right]^{2}} .
$$

Note that this expression originally derived for massive charge carriers with mobility $\mu=e \tau / m^{*}$ ( $\tau$ is the elastic scattering time and $m^{*}$ is the effective mass) is also valid for massless particles when defining the mobility more generally as the ratio between the average drift velocity of the carriers and the applied electric field. 

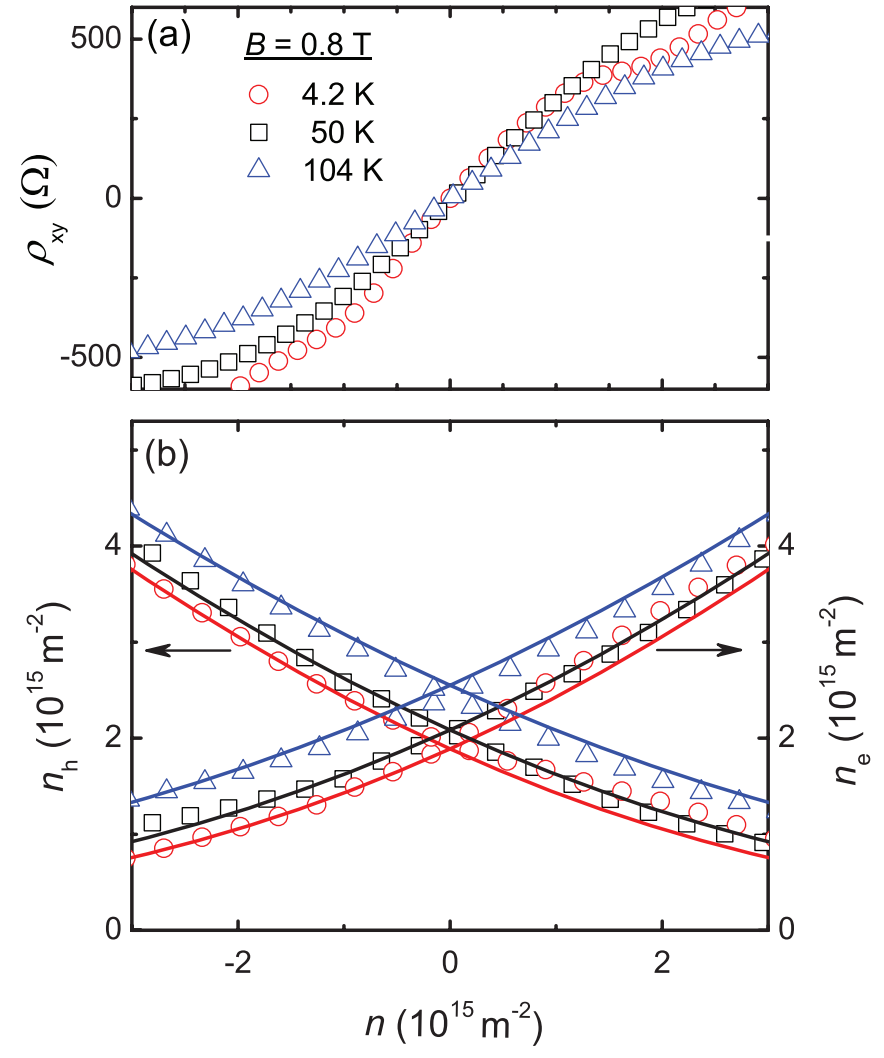

FIG. 1. (Color online) (a) Hall resistivity of the BLG sample as a function of the total charge carrier concentration at $B=0.8$ $\mathrm{T}$ for various temperatures. (b) Extracted carrier concentrations of electrons $n_{e}$ and holes $n_{h}$ for the same field and temperatures (see text for details). The solid lines show the calculated number of electrons and holes using the effective density of states from Eq. (4) and $s=$ $19 \mathrm{meV}$.

By solving the system of Eqs. (1) and (2) with respect to the variables $n_{e}$ and $n_{h}$ for each point of the experimental $\rho_{x y}\left(V_{g}\right)$ dependence, we can determine the number of electrons $n_{e}$ and holes $n_{h}$ independently; ${ }^{6}$ the results are shown in Fig. 1(b).

At the CNP $(n=0) n_{e}$ and $n_{h}$ are equal to a residual concentration $n_{0}$, which is, according to Eq. (2), related to the slope of $\rho_{x y}(n)$ at the CNP:

$$
\left.\frac{d \rho_{x y}}{d n}\right|_{n=0}=\frac{B}{e} \frac{1+(\mu B)^{2}}{4 n_{0}^{2}} .
$$

The temperature dependence of $n_{0}$ is plotted in Fig. 2(a). At zero temperature we find that a finite number of both electrons and holes are present at the CNP. These electron-hole puddles originate from spatial potential fluctuations within the sample represented schematically in Fig. 2(b) and lead to an effective density of states (DOS) around the CNP plotted in Fig. 2(c), ${ }^{9}$

$$
D(E)=D_{0} \operatorname{erfc}(-E / \sqrt{2} s) / 2,
$$

where $s$ is the amplitude of potential fluctuations.

Though the question of whether the puddles are caused by the impurity potential alone or by intrinsic ripples in graphene is still under discussion, ${ }^{10}$ this phenomenological DOS is not sensitive to the deeper origin of the puddles. The calculated charge carrier concentrations using this effective
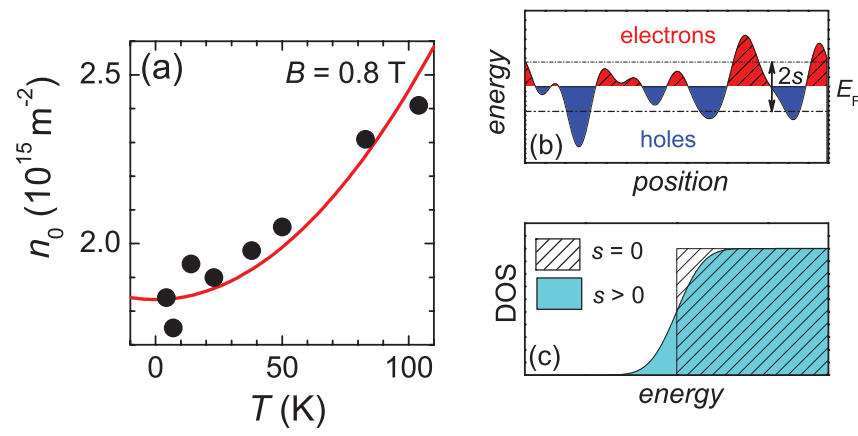

FIG. 2. (Color online) (a) Electron and hole concentration $n_{0}$ of the BLG sample at the CNP as a function of temperature. The solid line represents a fit to Eq. (5) with $s=19 \pm 2 \mathrm{meV}$. (b) Sketch of the random potential fluctuations in the sample yielding a broadened effective density of states shown in (c) (Ref. 9).

DOS, shown by the solid lines in Fig. 1(b), indeed reproduce the experimentally observed behavior around the CNP.

Directly at the CNP, the temperature dependence $n_{0}(T)$ in a low-temperature $\left(k_{B} T / s<1\right)$ approximation is analytically found to be ${ }^{9}$

$$
n_{0}(T)=n_{0}(0)\left[1+\frac{\pi^{2}}{6}\left(\frac{k_{B} T}{s}\right)^{2}\right]
$$

and a fit of the data in Fig. 2(a) to Eq. (5) yields $s=19 \pm$ $2 \mathrm{meV}$.

Analogously, the temperature dependence of the conductivity at the CNP can be described by ${ }^{9}$

$$
\sigma_{x x}(T)=\sigma_{x x}(0)\left[1+\sqrt{\frac{2}{\pi}} \frac{k T}{s}+\frac{\pi^{2}}{6}\left(\frac{k_{B} T}{s}\right)^{2}\right] .
$$

The fit of the experimental temperature dependence in the classical regime with Eq. (6) is shown in the inset of Fig. 3 for

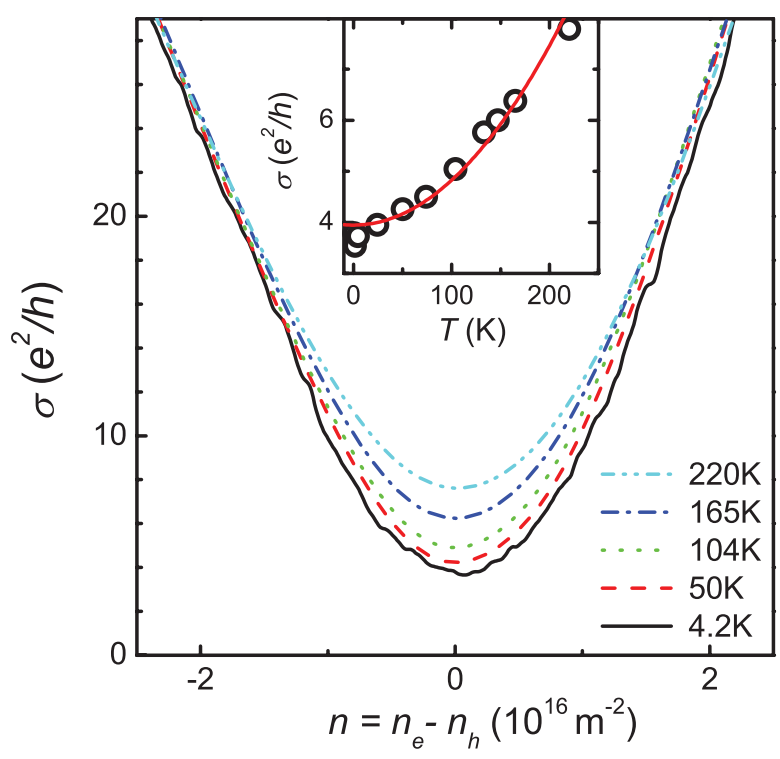

FIG. 3. (Color online) Zero-field conductivity of the BLG sample around the CNP for different temperature. The inset shows the temperature dependence of the minimum conductivity at the CNP with the solid line as a fit to Eq. (6) yielding $s=23 \pm 2 \mathrm{meV}$. 
the $0 \mathrm{~T}$ trace. The fit yields $s=23 \pm 2 \mathrm{meV}$, which is close to the value $s=19 \pm 2 \mathrm{meV}$, determined from the fit to the Hall data.

Remarkably, this strength of potential fluctuations is comparable to the thermal energy at room temperature and considerably larger than in conventional semiconductor heterostructures. ${ }^{11}$ Therefore, whereas quantum Hall localization in GaAs-based systems breaks down above $30 \mathrm{~K},{ }^{12}$ the strong potential fluctuations can induce a robust localization of charge carriers in graphene when the Fermi energy is situated between two Landau levels, in particular around filling factor $v=2{ }^{13}$ This allows the QHE to persist up to room temperature. $^{14,15}$

\section{QUANTIZED REGIME}

\section{A. Experimental results}

Figure 4(a) shows the measured Hall effect for BLG as a function of the total charge carrier concentration $n$ at $B=30 \mathrm{~T}$ for various temperatures. For $B \geqslant 15 \mathrm{~T}$ the system is in the quantized regime and we observe plateaus at $v= \pm 4, \pm 8 \ldots$ as expected for BLG. At the lowest temperatures, a plateau also develops at $v=-3$, which makes the $\rho_{x y}$ curve slightly asymmetric. However, since the plateau is smoothed out for $T>74 \mathrm{~K}$, we neglect this asymmetry in the following.

At $T=0.5 \mathrm{~K}$, the Hall resistance on the electron (hole) side reaches its maximum (minimum) value at the plateau and goes smoothly to the hole (electron) side as already observed in the nonquantized regime; this behavior can again be qualitatively explained by the two-carrier model. At high temperatures, the Hall resistivity overshoots the plateau $v= \pm 4$ before the beginning of the plateau and reaches values higher than $h / 4 e^{2}$ and $\rho_{x y}(n)$ becomes steeper at the CNP; see Fig. 4(a). We observe a similar behavior in the SLG sample, therefore this effect has a generic nature and was also observed by others. ${ }^{16}$ Note that these overshoots in the Hall resistance only appear at high temperatures and are therefore not due to the (partial) splitting of the lowest Landau level as observed in, e.g., Ref. 17.

The temperature dependence of the slope of $\rho_{x y}(n)$ at the CNP is explicitly shown in the inset to Fig. 4(a). It is opposite to the classical regime, which can be seen by comparison of the traces at $B=30 \mathrm{~T}$ (quantized regime) and $B=0.8 \mathrm{~T}$ (classical regime), plotted together in the inset.

According to Eq. (3), an increase in the slope with increasing temperature corresponds to a decrease of the charge carrier concentration, which appears to be counterintuitive. However, the plateaus remain visible up to the highest temperature measured, i.e., the system remains quantized and the number of electrons and holes within one Landau level is fixed. In the quantized regime, the slope of the Hall resistivity depends not only on the number of charge carriers at the CNP, but also the strength of localization, i.e., the width of the plateaus closest to the CNP. Therefore, Eq. (3) cannot be used to relate the slope to the number of charge carriers. In fact, Eq. (2), which predicts a $1 / n$ behavior of the slope around the CNP when $\mu B \rightarrow \infty$, anticipates such an overshoot.
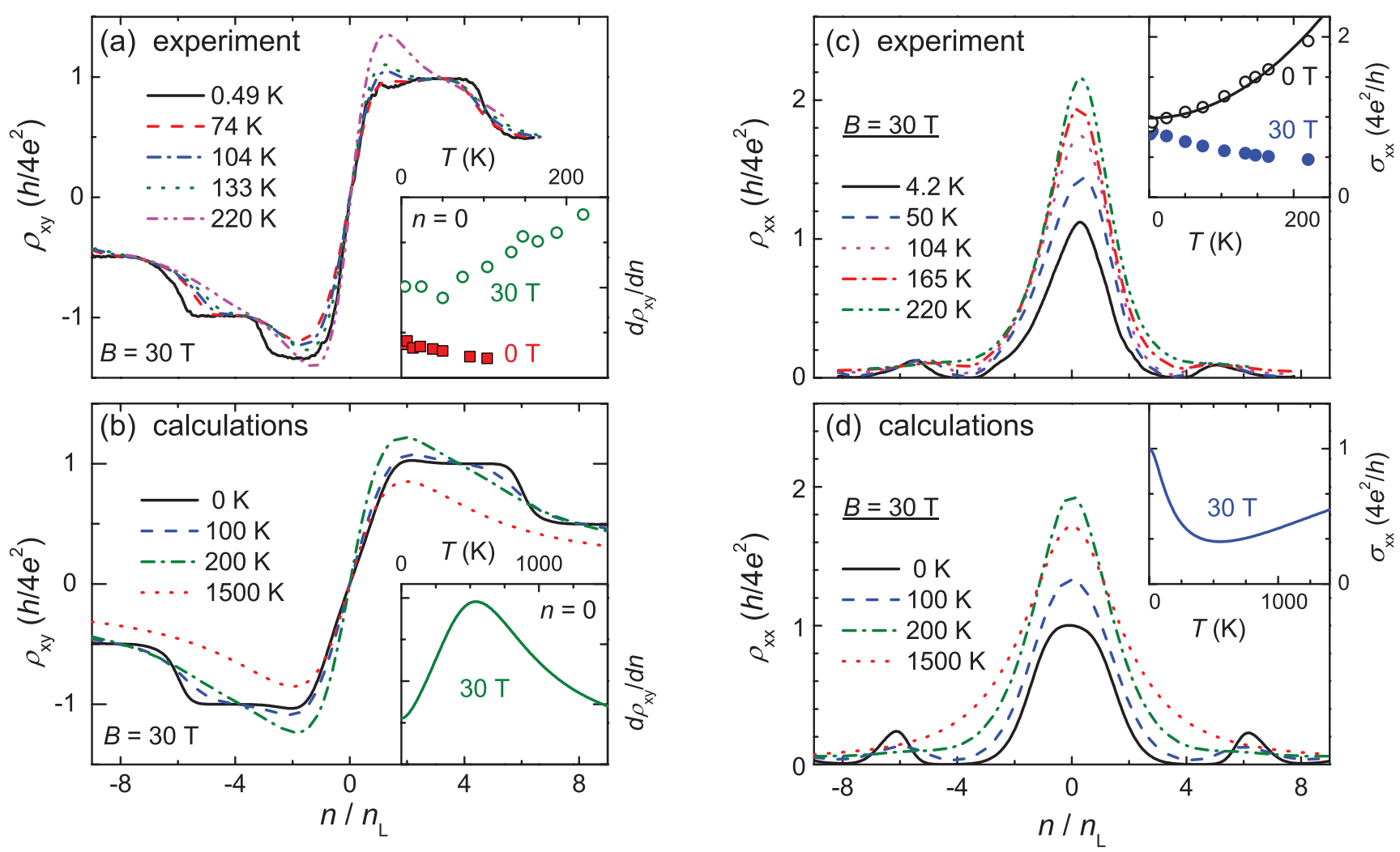

FIG. 4. (Color online) Transport coefficients $\rho_{x y}$ and $\rho_{x x}$ and of the BLG sample. Panel (a) and (c) show the experimentally measured values that are compared to the calculated quantities in (b) and (d). The insets in (a) and (c) show the corresponding slopes of the Hall resistivity at the CNP as a function of temperature. The insets in (b) and (d) show the temperature dependence of the conductivity at the CNP. 
A similarly unexpected behavior for high temperature is observed in the resistance at high magnetic fields. Figure 4(c) shows the resistivity of BLG in the quantized regime as a function of the total charge carrier concentration for various temperatures. In contrast to the nonquantized regime, the resistivity exhibits a metal-like increase with temperature.

As we will show in the following section, this peculiar transport properties in the quantized regime can be described in a simple DOS model with localized and extended states where the temperature dependence of the resistivity is governed by a redistribution of charge carriers due to the thermal activation.

\section{B. Model calculations and discussion}

The model used for our calculation starts with the quantized DOS for graphene in magnetic field consists of separate Gaussian-shaped Landau levels (LL's) as depicted schematically in Fig. 5. States in the middle of each LL are extended, and states are localized in the tails. For our simulations of the DOS in BLG shown below, we have used a constant width $\Delta=100 \mathrm{~K}$ for the higher Landau levels and $\Delta=200 \mathrm{~K}$ for the zero-energy LL. ${ }^{18}$ Landau levels above (below) the CNP, populated by electrons (holes), have a degeneracy of $4 e B / h$. The zeroth LL with a degeneracy of $4 e B / h$ for SLG and $8 e B / h$ for BLG is populated by electrons and holes simultaneously, such that electron and hole conduction can be found both above and below the CNP. We can then calculate the conductivity using the Kubo-Greenwood formalism: ${ }^{19,20}$

$$
\begin{aligned}
\sigma_{x x} & =\sigma_{x x}^{e}+\sigma_{x x}^{h} \\
& =\int_{-\infty}^{\infty}\left[K^{e}(E) \frac{\partial f(E)}{\partial E}+K^{h}(E) \frac{\partial[1-f(E)]}{\partial E}\right] d E .
\end{aligned}
$$

Here $f(E)$ is the Fermi distribution and $K^{e, h}(E)$ is an energydependent function containing the density of the extended states, the particle velocity, and the scattering time. The upper indices $e, h$ correspond to electrons and holes, respectively. Modeling $K(E)$ by a superposition of Gaussians with the above-mentioned widths, the Kubo-Greenwood formula reproduces qualitatively the behavior of the experimental conductivity in graphene, but does not give a universal value of minimal conductivity. ${ }^{21}$ Therefore, we normalized the integral in Eq. (7) such that at zero temperature, $\sigma_{x x}$ at the CNP has the universal value of $4 e^{2} / h$ in accordance with the experimentally observed value.

Similarly, we can determine the Hall conductivity by summing up all extended states below the Fermi level, smeared

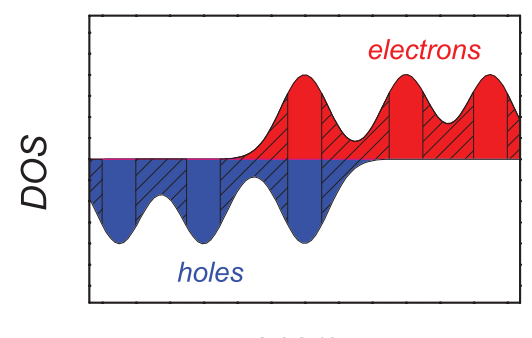

energy

FIG. 5. (Color online) Schematic density of states (DOS) of broadened Landau levels in BLG. The dashed regions correspond to localized states between two levels. out by temperature:

$$
\sigma_{x y} \propto \int_{-\infty}^{\infty}\left\{D_{\text {ext }}^{h}[1-f(E)]-D_{\text {ext }}^{e} f(E)\right\} d E .
$$

When introducing a localized DOS, the calculated conductivity $\sigma_{x y}$ develops plateaus that are not at multiples of $4 e^{2} / h,{ }^{22}$ because the number of extended states within a LL is less than the total level degeneracy. In order to reproduce the correct number of occupied LL's in Eq. (8), we have normalized the extended DOS for each LL to the total number of extended states within it.

After calculating the conductivities, we convert them into the resistivity $\rho_{x x}$ and the Hall resistivity $\rho_{x y}$ using the standard matrix relations; the results are shown in Fig. 4(b) and 4(d). They can be compared to the experimental curves, plotted as panels (a) and (c) of the figures. The calculated curves reproduce qualitatively the experimental results and display a similar temperature dependence. In particular, at the CNP, the resistivity [insets in Figs. 4(c) and 4(d)] and the slope of the Hall resistivity [insets in Figs. 4(a) and 4(b)] increase with increasing temperature up to $200 \mathrm{~K}$. Moreover, as is also seen in the experiment, the overshoots develop at the beginning of the plateaus at high temperatures [compare panels (a) and (b) in Fig. 4].

Starting from about $500 \mathrm{~K}$ (as calculated for $30 \mathrm{~T}$ ) the system smoothly enters the classical regime. The conductivity [inset to Fig. 4(d)] starts to increase with increasing temperature, and the slope of the Hall resistivity [inset to Fig. 4(b)] decreases. Experimentally, this trend in the nonquantized regime is verified by the low field data presented earlier in Figs. 2 and 3.

\section{CONCLUSIONS}

To conclude, we have investigated the coexistence of electron and hole magnetotransport in graphene for a wide range of temperatures and magnetic fields. For all temperatures and fields, the Hall resistivity smoothly crosses zero at the CNP, indicating the simultaneous presence of both electrons and holes at the CNP. In the nonquantized regime, the slope of the Hall resistivity decreases with increasing temperature, which is accompanied by an increasing conductivity at the CNP. This behavior can be quantitatively modeled by a thermal activation of charge carriers experiencing relatively large potential fluctuation of the order of $20 \mathrm{meV}$.

In the quantum Hall regime, increasing the temperature has the opposite effect on the slope of the Hall effect and produces counterintuitive overshoots when approaching the $v= \pm 2$ plateaus. Using an appropriate density of states, we have shown in model calculations that such a behavior is expected for any two-carrier system with a quantized energy spectrum.

\section{ACKNOWLEDGMENTS}

Part of this work has been supported by EuroMagNET under EU Contract No. 228043 and by the Stichting Fundamenteel Onderzoek der Materie (FOM) with financial support from the Nederlandse Organisatie voor Wetenschappelijk Onderzoek (NWO). 
*E.Kurganova@science.ru.nl

†U.Zeitler@ science.ru.nl

${ }^{1}$ Y.-W. Tan, Y. Zhang, K. Bolotin, Y. Zhao, S. Adam, E. H. Hwang, S. Das Sarma, H. L. Stormer, and P. Kim, Phys. Rev. Lett. 99, 246803 (2007).

${ }^{2}$ Y. Zhang, V. W. Brar, C. Girit, A. Zettl, and M. F. Crommie, Nat. Phys. 5, 722 (2009).

${ }^{3}$ J. Martin, N. Akerman, G. Ulbricht, T. Lohmann, J. H. Smet, K. von Klitzing, and A. Yacoby, Nat. Phys. 4, 144 (2008).

${ }^{4}$ G. M. Rutter, S. Jung, N. N. Klimov, D. B. Newell, N. B. Zhitenev, and J. A. Stroscio, Nat. Phys. 7, 649 (2011).

${ }^{5}$ K. S. Novoselov, A. K. Geim, S. V. Morozov, D. Jiang, Y. Zhang, S. V. Dubonos, I. V. Grigorieva, and A. A. Firsov, Science 306, 666 (2004).

${ }^{6}$ S. Wiedmann, H. J. van Elferen, E. V. Kurganova, M. I. Katsnelson, A. J. M. Giesbers, A. Veligura, B. J. van Wees, R. V. Gorbachev, K. S. Novoselov, J. C. Maan, and U. Zeitler, Phys. Rev. B 84, 115314 (2011).

${ }^{7}$ N. W. Ashcroft and N. D. Mermin, Solid State Physics (Saunders College, Philadelphia, 1976), p. 240.

${ }^{8}$ E. H. Hwang, S. Adam, and S. Das Sarma, Phys. Rev. B 76, 195421 (2007).

${ }^{9}$ E. H. Hwang and S. Das Sarma, Phys. Rev. B 82, 081409 (2010).

${ }^{10}$ M. Gibertini, A. Tomadin, F. Guinea, M. I. Katsnelson, and M. Polini, Phys. Rev. B 85, 201405 (2012).

${ }^{11}$ J. A. Nixon and J. H. Davies, Phys. Rev. B 41, 7929 (1990).
${ }^{12}$ L. B. Rigal, D. K. Maude, M. Potemski, J. C. Portal, L. Eaves, Z. R. Wasilewski, G. Hill, and M. A. Pate, Phys. Rev. Lett. 82, 1249 (1999).

${ }^{13}$ A. J. M. Giesbers, U. Zeitler, M. I. Katsnelson, L. A. Ponomarenko, T. M. Mohiuddin, and J. C. Maan, Phys. Rev. Lett. 99, 206803 (2007).

${ }^{14}$ K. S. Novoselov, Z. Jiang, Y. Zhang, S. V. Morozov, H. L. Stormer, U. Zeitler, J. C. Maan, G. S. Boebinger, P. Kim, and A. K. Geim, Science 315, 1379 (2007).

${ }^{15}$ The recent observation of a robust quantum Hall effect in InSb systems (Ref. 23) may indicate that such a robust localization can also be achieved in conventional semiconductor heterojunctions.

${ }^{16}$ Z. Jiang, Y. Zhang, Y.-W. Tan, H. Stormer, and P. Kim, Solid State Commun. 143, 14 (2007).

${ }^{17}$ Z. Jiang, Y. Zhang, H. L. Stormer, and P. Kim, Phys. Rev. Lett. 99, 106802 (2007).

${ }^{18}$ E. V. Kurganova, A. J. M. Giesbers, R. V. Gorbachev, A. K. Geim, K. S. Novoselov, J. C. Maan, and U. Zeitler, Solid State Commun. 150, 2209 (2010).

${ }^{19}$ R. Kubo, Can. J. Phys. 34, 1274 (1956).

${ }^{20}$ D. A. Greenwood, Proc. Phys. Soc. London 71, 585 (1958).

${ }^{21}$ K. Ziegler, Phys. Rev. B 75, 233407 (2007).

${ }^{22}$ K. von Klitzing, Rev. Mod. Phys. 58, 519 (1985).

${ }^{23}$ J. A. Alexander-Webber, A. M. R. Baker, P. D. Buckle, T. Ashley, and R. J. Nicholas, Phys. Rev. B 86, 045404 (2012). 